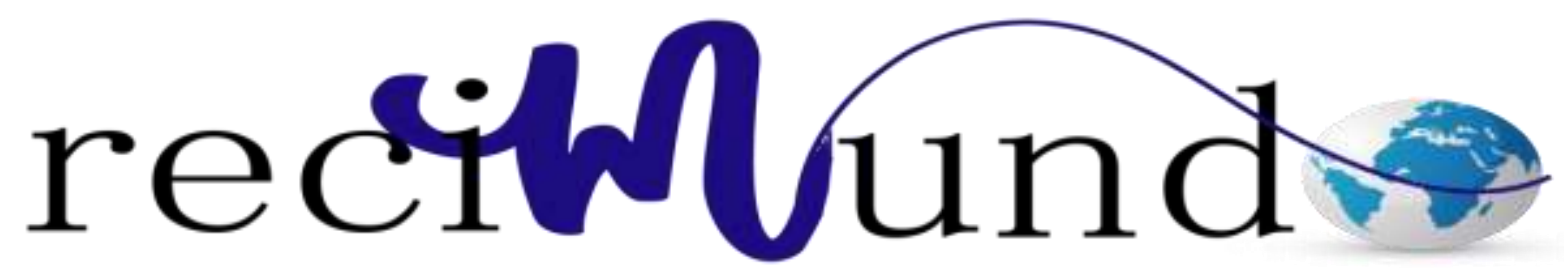

Revista Cientifica Mundo de la Investigación y el Conocimiento

Gabriela Rosa Rodríguez Plaza a ; Néstor Ricardo Espinosa Sánchez ${ }^{\text {b }}$, Renato Paolo

Patiño Andrade ${ }^{c}$; Marlon José Rivadeneira Rodríguez ${ }^{\mathrm{d}}$

Cristaloides y coloides en la reanimación del paciente crítico

Crystaloids and coloids in the reusimation of the critical patient

Revista Científica Mundo de la Investigación y el Conocimiento. Vol. 3 núm.3, septiembre, ISSN: 2588-073X, 2019, pp. 372-392

DOI: $10.26820 /$ recimundo/3.(3).septiembre.2019.372-392

URL: http://recimundo.com/index.php/es/article/view/526

Código UNESCO: 3205 Medicina Interna

Tipo de Investigación: Artículo de Revisión

Editorial Saberes del Conocimiento

Recibido: 15/05/2019

Aceptado: 23/06/2019

Publicado: 30/09/2019

Correspondencia: shirleyvi16co@ hotmail.com

a. Médico; Investigador Independiente; Guayaquil, Ecuador; doctora.gabriela.gr@gmail.com

b. Médico; Investigador Independiente; Guayaquil, Ecuador; drnespinosa@ hotmail.com

c. Médico; Investigador Independiente; Guayaquil, Ecuador; polo_rp69@ hotmail.com

d. Médico; Investigador Independiente; Guayaquil, Ecuador; marlon.riro94@gmail.com 


\section{Cristaloides y coloides en la reanimación del paciente crítico}

Vol. 3, núm. 3., (2019)

Gabriela Rosa Rodríguez Plaza; Néstor Ricardo Espinosa Sánchez; Renato Paolo Patiño

Andrade; Marlon José Rivadeneira Rodríguez

\section{RESUMEN}

Los escenarios clínicos más frecuentes a los que se enfrenta el profesional de la salud de manera continua en el área de urgencias, hospitalización, Unidad de Terapia Intensiva (UTI), quirófano, entre otras, son el estado de choque hipovolémico, séptico y cardiogénico, que dependiendo de su etiología, fisiopatología y comportamiento hemodinámico, se llevarán diferentes intervenciones con el objetivo de estabilizar al enfermo. El punto clave, para una reanimación exitosa en el enfermo grave, es llevarla a cabo de manera temprana y oportuna una vez detectado el proceso desencadenante, cumpliendo con las metas y tiempo estipulados para ello, lo que se verá reflejado en una disminución de la morbilidad y mortalidad de los pacientes. En base a esto, se presenta la reanimación con fluidos, la cual representa una herramienta esencial para la supervivencia del paciente crítico en shock, independientemente de la causa que lo origine. Hoy en día se dispone de diversos cristaloides y coloides (sintéticos y naturales), existiendo una viva controversia sobre qué tipo de fluidos que deben ser empleados para tal fin, y los posibles efectos adversos asociados a su uso, especialmente el desarrollo de fracaso renal con necesidad de técnicas de reemplazo renal. Estas técnicas representan la piedra angular para mejorar la sobrevivencia de los pacientes en estado crítico, pues constituyen proceso de reanimación, que debe realizarse de manera temprana, oportuna y dirigida por metas. La terapia intravenosa a utilizarse debe ser individualizada dependiendo de la causa de morbilidad del enfermo, ya que de manera general no ha demostrado ser superior la administración de coloide sobre cristaloides. El objetivo de este artículo es llevar a cabo una revisión sistemática de la literatura, con análisis crítico de la misma, con la finalidad de proporcionar al lector literatura de importancia sobre este tema.

Palabras Claves: Cristaloides; Coloides; Reanimación del paciente Crítico; Estado de Choque; Proceso de Reanimación; Terapia Intravenosa. 


\title{
Cristaloides y coloides en la reanimación del paciente crítico
}

Vol. 3, núm. 3., (2019)

Gabriela Rosa Rodríguez Plaza; Néstor Ricardo Espinosa Sánchez; Renato Paolo Patiño Andrade; Marlon José Rivadeneira Rodríguez

\begin{abstract}
The most frequent clinical scenarios that the healthcare professional faces continuously in the emergency department, hospitalization, Intensive Care Unit (ICU), operating room, among others, are the state of hypovolemic, septic and cardiogenic shock, that depending on its etiology, pathophysiology and hemodynamic behavior, different interventions will be carried out in order to stabilize the patient. The key point, for a successful resuscitation in the seriously ill patient, is to carry it out early and in a timely manner once the triggering process has been detected, complying with the goals and time stipulated for it, which will be reflected in a decrease in morbidity and patient mortality. Based on this, the resuscitation with fluids is presented, which represents an essential tool for the survival of the critical patient in shock, regardless of the cause that causes it. Today there are several crystalloids and colloids (synthetic and natural), there is a lively controversy about what kind of fluids that should be used for this purpose, and the possible adverse effects associated with their use, especially the development of renal failure with Need for renal replacement techniques. These techniques represent the cornerstone to improve the survival of critically ill patients, as they constitute a resuscitation process, which must be carried out early, in a timely manner and directed by goals. The intravenous therapy to be used should be individualized depending on the cause of morbidity of the patient, since in general the administration of colloid on crystalloids has not been shown to be superior. The objective of this article is to carry out a systematic review of the literature, with a critical analysis of it, in order to provide the reader with important literature on this subject.
\end{abstract}

Key Words: Crystalloids; Colloids; Critical Patient Resuscitation; Shock Status; Resuscitation Process; Intravenous Therapy. 


\section{Cristaloides y coloides en la reanimación del paciente crítico}

Vol. 3, núm. 3., (2019)

Gabriela Rosa Rodríguez Plaza; Néstor Ricardo Espinosa Sánchez; Renato Paolo Patiño

Andrade; Marlon José Rivadeneira Rodríguez

\section{Introducción.}

La administración de fluidos es uno de los actos terapéuticos que con mayor frecuencia realizan en su práctica asistencial cotidiana los médicos que atienden a pacientes críticamente enfermos. Este aporte ocurre en mayor cuantía durante las primeras horas y días de estancia, dado que durante las mismas es cuando se lleva a cabo la reanimación del paciente que con frecuencia ingresa en UCI por shock o hipotensión de cualquier etiología. No obstante, se debe tener muy presente que los fluidos deben ser administrados con la misma precaución e igual grado de conocimiento (indicaciones, contraindicaciones, efectos adversos) que se tiene para cualquier fármaco. (Rozoli, 2015).

En este contexto, hay factores que son esenciales, los cuales se plantean los clínicos a diario y se reflejan en las hipótesis de trabajo de los diversos estudios y ensayos clínicos. Estas cuestiones son: ¿qué fluido aportar? y ¿cuánto fluido administrar y en cuánto tiempo?. En torno a esto, se debe resaltar que existen nuevas soluciones disponibles en el mercado, así como reciente información sobre la adecuación e idoneidad de las diversas soluciones en diferentes escenarios clínicos. Estos nuevos datos son a veces contradictorios, faltando en muchos casos conclusiones definitivas. Todo ello explica la gran variedad de prescripciones de fluidos que existe, dado que hay países en los que el empleo de coloides es casi anecdótico, y otros donde constituyen la primera línea de tratamiento para la hipotensión.

Actualmente, este tema está siendo muy discutido y controvertido; de hecho, se ha originado un reciente consenso auspiciado por la ESICM, el cual considera que no deben utilizarse los coloides sintéticos en pacientes críticos fuera del marco de proyectos de 


\section{Cristaloides y coloides en la reanimación del paciente crítico}

Vol. 3, núm. 3., (2019)

Gabriela Rosa Rodríguez Plaza; Néstor Ricardo Espinosa Sánchez; Renato Paolo Patiño Andrade; Marlon José Rivadeneira Rodríguez

investigación Ello hace que solo queden disponibles los cristaloides y la albúmina. No obstante, el uso exclusivo de cristaloides no está exento de riesgo, en especial el desarrollo de edema intersticial, el cual es reconocido bajo el término de cristaloide, en donde se engloban diversas soluciones con composiciones diferentes.

En el año 2001, Rivers evaluó un proceso de reanimación dirigido por metas en pacientes con sepsis en el departamento de urgencias, demostrando una disminución de la mortalidad del $16 \%$ en los enfermos que cumplieron con los objetivos dentro de las primeras seis horas. Tal fue el impacto de este estudio, que ha sido tomado como modelo para lo toma de decisiones en los procesos de reanimación y aplicado a otras entidades clínicas en las diferentes áreas críticas hospitalarias, lo que ha llevado a que parte fundamental en el proceso de reanimación es decidir el tipo de terapia intravenosa para reposición de volumen a emplear, coloide o cristaloide. De ahí, que los estudios clínicos que se han realizado a través del tiempo, han surgido con la finalidad de demostrar la superioridad de uno sobre el otro, o viceversa. (Beck, 2016).

La mayoría de los médicos de los países desarrollados hoy en día empiezan la reanimación de los pacientes traumatizados de acuerdo a las guías del ATLS (Advanced Trauma Life Support) del Colegio Americano de Cirujanos. Las guías actuales muestran un régimen agresivo de reanimación con líquidos que inicia con un bolo de 2 litros de cristaloides, preferiblemente Lactato de Ringer (LR). La reanimación continúa con bolos repetidos de LR, sangre y una búsqueda sistemática y reparación quirúrgica de las fuentes hemorrágicas corregibles (Lang, 2015). 


\section{Cristaloides y coloides en la reanimación del paciente crítico}

Vol. 3, núm. 3., (2019)

Gabriela Rosa Rodríguez Plaza; Néstor Ricardo Espinosa Sánchez; Renato Paolo Patiño Andrade; Marlon José Rivadeneira Rodríguez

Tabla $\mathbf{N}^{\circ}$ 1. Complicaciones Asociadas con el uso de Cristaloides

\begin{tabular}{|c|c|}
\hline Soluciōn Cristaloide & Posible Alteración \\
\hline $\begin{array}{l}\text { Lactato de Ringer } \\
\text { Solución Hartmann }\end{array}$ & $\begin{array}{l}\text { - Hipercaliemia en pacientes renales con infusiones altag } \\
\text { - Alcalosis metabólica por que el lactsto metaboliza a bicar- } \\
\text { bonato en el higalosi }\end{array}$ \\
\hline $\begin{array}{l}\text { Solución Salina } \\
\text { Normal }\end{array}$ & $\begin{array}{l}\text { - Hipercloremia con altas infusiones en pacientes con deterioro } \\
\text { de su función renal. } \\
\text { - Acidosis hiperclorémica } \\
\text { (5) }\end{array}$ \\
\hline $\begin{array}{l}\text { Solución Salina } \\
\text { Hipertônica }\end{array}$ & $\begin{array}{l}\text { - Hipernatremia(w) } \\
\text { - Acidosis metabólica }{ }^{(4)} \\
\text { - Hipocaliemia } \\
\text { - Deshidratación cerebral con sangrado intracraneano } \\
\text { - Mielinolisis póntica por cambios abruptos en la concentración } \\
\text { de sodió(t) } \\
\text { - Edema pulmonar en pacientes con cardiopatias }\end{array}$ \\
\hline $\begin{array}{l}\text { Dextrosas } \\
\text { (soluciones } \\
\text { glucosadas) }\end{array}$ & $\begin{array}{l}\text { - Intoxicación acuosa por una sobrecarga de la solución glu- } \\
\text { cosadact. } \\
\text { - Hiperglicemia } \\
\text { - Pacientes con enfermedad de Adisson: crisis addisoniana. } \\
\text { - Deshidratación celular }\end{array}$ \\
\hline
\end{tabular}

Fuente: (Lagn 2015).

Los cristaloides primariamente llenan el espacio intersticial, por eso el edema es un resultado esperado de la reanimación de acuerdo a las guías del ATLS. Para expandir el volumen plasmático se usa el axioma clásico de utilizar tres veces más el volumen de cristaloides por volumen de sangre perdida. Hoy, esta relación es cuestionada, probablemente sea 7:1 o 10:1 por la disminuida presión coloido-osmótica secundaria a la disminución de la concentración sérica de las proteínas por la hemorragia, fuga capilar y reemplazo con cristaloides. (Lang 2015).

Una vez el médico considere utilizar coloides, el paso a seguir es escoger entre las diferentes soluciones. (Avalo 2017). El prototipo de coloide es la albúmina, sintetizada en el 


\section{Cristaloides y coloides en la reanimación del paciente crítico}

Vol. 3, núm. 3., (2019)

Gabriela Rosa Rodríguez Plaza; Néstor Ricardo Espinosa Sánchez; Renato Paolo Patiño Andrade; Marlon José Rivadeneira Rodríguez

hígado y responsable del $80 \%$ de la presión oncótica del plasma. Las desventajas frecuentemente mencionadas incluyen el hecho de ser hemoderivado, el riesgo teórico de transmitir partículas desconocidas infecciosas (priones), y como los otros coloides, su alto costo. (Brattong, 2016). De los coloides sintéticos, las gelatinas y los dextranos, son considerados rara vez para la reanimación del paciente traumatizado, especialmente en Norteamérica. Aparte de sus propiedades expansoras plasmáticas, los dextranos tienen un poderoso efecto anticoagulante, y ha sido para la prevención de la trombosis venosa postoperatoria y el embolismo pulmonar. (Lang 2015).

Las propiedades anticoagulantes de los dextranos han limitado sus indicaciones en la reanimaron de pacientes traumatizados. Sin embargo, pequeñas cantidades han sido utilizadas para prolongar el efecto de la infusión de solución salina hipertónica. Las gelatinas son las menos disponibles en Norteamérica y su corta vida de efectividad ha disminuido el entusiasmo por este tipo de coloide en el ámbito mundial. A tal efecto, se debe resaltar que varias soluciones coloides han sido desarrolladas y usadas en la práctica clínica a nivel mundial. Su uso requiere del conocimiento de las propiedades químicas de los coloides y las características únicas de cada solución.

Los coloides son costosos y presentan reacciones alérgicas y efectos adversos coagulopáticos que no son vistos con los cristaloides. Además algunas patologías críticas, cursan con aumento de la permeabilidad vascular, lo cual limita la habilidad de los coloides para mantener el espacio vascular y ejercer una presión osmótica adicional. (Nicholson, 2016). La fuga vascular aumenta el coloide en el espacio intersticial, llevando a la formación de edemas. A 


\section{Cristaloides y coloides en la reanimación del paciente crítico}

Vol. 3, núm. 3., (2019)

Gabriela Rosa Rodríguez Plaza; Néstor Ricardo Espinosa Sánchez; Renato Paolo Patiño

Andrade; Marlon José Rivadeneira Rodríguez

pesar de algunas ventajas teóricas comparadas con los cristaloides, la administración de coloides no ha mostrado disminución del riesgo de injuria pulmonar aguda, o mejoría de la supervivencia.

Las indicaciones precisas del uso de soluciones coloides incluyen estados hipoproteinémicos, pacientes que requieren expansión plasmática y no son capaces de tolerar grandes cantidades de volumen, procedimientos reconstructivos y ortopédicos que requieran prevención de formación de trombos y leucoféresis Contrario a esto, las soluciones de cristaloides isotónicas respecto al plasma se van a distribuir por el fluido extracelular, estos fluidos presentan un alto índice de eliminación y se puede estimar que, en voluntarios sanos, a los 60 min de la administración permanece solo el $20 \%$ del volumen infundido en el espacio intravascular La solución salina al 0,9\%, también denominada suero fisiológico, es levemente hipertónica respecto al líquido extracelular y tiene un pH ácido. (Moss, 2017)

Tipo de investigación

Dentro de toda práctica investigativa, se precisan acciones de carácter metodológico mediante las cuales, se logra conocer y proyectar los eventos posibles que la determinan, así como las características que hacen del acto científico un proceso interactivo ajustado a una realidad posible de ser interpretada. En este sentido, se puede decir, que la presente investigación corresponde al tipo documental, definido por (Dávila, 2012), "se ocupa del estudio de problemas planteados a nivel teórico, la información requerida para abordarlos se encuentra básicamente en materiales impresos, audiovisuales y /o electrónicos”. (p.41).

En consideración a esta definición, la orientación metodológica permitió la oportunidad de cumplir con una serie de actividades inherentes a la revisión y lectura de diversos documentos 


\section{Cristaloides y coloides en la reanimación del paciente crítico}

Vol. 3, núm. 3., (2019)

Gabriela Rosa Rodríguez Plaza; Néstor Ricardo Espinosa Sánchez; Renato Paolo Patiño Andrade; Marlon José Rivadeneira Rodríguez

donde se encontraron ideas explicitas relacionadas con los tópicos encargados de identificar a cada característica insertada en el estudio. Por lo tanto, se realizaron continuas interpretaciones con el claro propósito de revisar aquellas apreciaciones o investigaciones propuestas por diferentes investigadores, para luego dar la respectiva argumentación a los planteamientos, en función a las necesidades encontradas en la indagación.

\section{Fuentes documentales}

El análisis correspondiente a las características que predomina en el tema seleccionado, llevan a incluir diferentes fuentes documentales encargadas de darle el respectivo apoyo y en ese sentido cumplir con la valoración de los hechos a fin de generar nuevos criterios que sirven de referencia a otros procesos investigativos. Para (Arias, 2010), las fuentes documentales incorporadas en la investigación documental o bibliográfica, "representa la suma de materiales sistemáticos que son revisados en forma rigurosa y profunda para llegar a un análisis del fenómeno". (p.41). Por lo tanto, se procedió a cumplir con la realización de una lectura previa determinada por encontrar aquellos aspectos estrechamente vinculados con los “CRISTALOIDES Y COLOIDES EN LA REANIMACIÓN DEL PACIENTE CRÍTICO” para luego explicar mediante un desarrollo las respectivas apreciaciones generales de importancia.

\section{Técnicas para la Recolección de la Información}

La conducción de la investigación para ser realizada en función a las particularidades que determinan a los estudios documentales, tiene como fin el desarrollo de un conjunto de acciones encargadas de llevar a la selección de técnicas estrechamente vinculadas con las características 


\section{Cristaloides y coloides en la reanimación del paciente crítico}

Vol. 3, núm. 3., (2019)

Gabriela Rosa Rodríguez Plaza; Néstor Ricardo Espinosa Sánchez; Renato Paolo Patiño

Andrade; Marlon José Rivadeneira Rodríguez

del estudio. En tal sentido, (Arias Ob cit) refiere, que es "una técnica particular para aportar ayuda a los procedimientos de selección de las ideas primarias y secundarias”. (p. 71).

Por ello, se procedió a la utilización del subrayado, resúmenes, fichaje, como parte básica para la revisión y selección de los documentos que presentan el contenido teórico. Es decir, que mediante su aplicación de estas técnicas se pudo llegar a recoger informaciones en cuanto a la revisión bibliográfica de los diversos elementos encargados de orientar el proceso de investigación. Tal como lo expresa, (Arias Ob cit) "las técnicas documentales proporcionan las herramientas esenciales y determinantes para responder a los objetivos formulados y llegar a resultados efectivos" (p. 58). Es decir, para responder con eficiencia a las necesidades investigativas, se introdujeron como técnica de recolección el método inductivo, que hizo posible llevar a cabo una valoración de los hechos de forma particular para llegar a la explicación desde una visión general.

Asimismo, se emplearon las técnicas de análisis de información para la realización de la investigación que fue ejecutada bajo la dinámica de aplicar diversos elementos encargados de determinar el camino a recorrer por el estudio, según, (Arias, Ob cit) las técnicas de procesamiento de datos en los estudios documentales "son las encargadas de ofrecer al investigador la visión o pasos que debe cumplir durante su ejercicio, cada una de ellas debe estar en correspondencia con el nivel a emplear" (p. 123). Esto indica, que para llevar a cabo el procesamiento de los datos obtenidos, es necesario establecer las técnicas que serán seleccionadas, destacándose en este caso, de manera particular: fichas de resumen, textual, registros descriptivos entre otros, los mismos se deben ajustar al nivel que ha sido seleccionado. 


\section{Cristaloides y coloides en la reanimación del paciente crítico}

Vol. 3, núm. 3., (2019)

Gabriela Rosa Rodríguez Plaza; Néstor Ricardo Espinosa Sánchez; Renato Paolo Patiño Andrade; Marlon José Rivadeneira Rodríguez

\section{Resultados.}

\section{Aplicación Clínica de Coloides y Cristaloides}

En la actualidad, existe evidencia contundente sobre el uso de los coloides versus cristaloides en pacientes graves; sin embargo, poseen importantes diferencias en cuanto a seguridad y eficacia. Los coloides ofrecen importantes ventajas teóricas sobre los cristaloides en el proceso de reanimación, pero algunos coloides (ej. hidroxietil almidón, dextranos) pueden presentar efectos adversos serios y, la administración de albúmina implica altos costos. El estudio SAFE (Saline versus Albumin Fluid Evaluation) comparó el efecto de la administración de albúmina $4 \%$ versus solución salina $0.9 \%$ en el proceso de reanimación en pacientes en la UTI. (Myllan, 2014).

El riesgo relativo (RR) de muerte fue de 0.99 (IC 95\% 0.91-1.09, p: 0.87) en el grupo de albúmina versus el grupo de solución salina. La mortalidad a los 28 días fue de 3.2\% en contra del $2.5 \%$ en el grupo de albúmina y solución salina respectivamente (RR 1.27, p: 0.09) de los pacientes que permanecieron en la UTI y, del 22.8 versus 24.5\% (RR 0.93 IC 95\% 0.86-1.01; p: 0.10) de los que permanecieron en hospitalización. El RR de mortalidad a los 28 días en los pacientes con trauma en el grupo de albúmina versus el grupo de solución salina fue del 1.36 (IC 95\% 0.99-1.86, p: 0.06), comparado con los pacientes sin trauma fue del 0.96 (p: 0.04). (Myllam 2014).

En los enfermos con sepsis grave el RR de muerte a los 28 días en el grupo de albúmina versus solución salina fue de 0.87 (IC 95\% 0.74-1.02, p: 0.09) comparado con los pacientes sin sepsis grave que fue de 1.05 (p: 0.06). En los pacientes con síndrome de insuficiencia respiratoria 


\section{Cristaloides y coloides en la reanimación del paciente crítico}

Vol. 3, núm. 3., (2019)

Gabriela Rosa Rodríguez Plaza; Néstor Ricardo Espinosa Sánchez; Renato Paolo Patiño

Andrade; Marlon José Rivadeneira Rodríguez

aguda (SIRA) el RR en pacientes en el grupo de albúmina versus solución salina fue de 0.93 , comparado con los pacientes sin SIRA de 1 (p: 0.74). En los pacientes con traumatismo craneoencefálico (TCE), la terapia intravenosa en el proceso de reanimación es parte fundamental para la restauración y mantenimiento de la circulación sistémica y cerebral. (Myburg, 2017).

Por lo anterior, el grupo de autores del estudio SAFE llevó a cabo un análisis post hoc de los pacientes con TCE (SAFE-TBI study) para determinar las causas de muerte y funcionalidad neurológica a 24 meses posteriores a la aleatorización. La mortalidad a los 24 meses fue de $33.2 \%$ en el grupo de albúmina comparado con el $20.4 \%$ en el grupo de solución salina (RR 1.63 IC 95\% 1.17-2.26; p: 0.003), ocurriendo dentro de los primeros 28 días hasta el $85 \%$ en ambos grupos. La mortalidad en los pacientes con TCE severo (Glasgow 3-8) fue de $41.8 \%$ en el grupo de albúmina versus $22.2 \%$ en el grupo de solución salina (RR 1.88, IC 95\% 1.31-2.70; p: < 0.001). (Myburg 2017)

Con relación al pronóstico neurológico, fue más favorable para el grupo de solución salina $60.6 \%$ versus el grupo de albúmina del $47.3 \%$ (RR 0.78, IC 95\% 0.65-0.94; p: 0.007).4 La sepsis se ha convertido en la principal causa de ingreso y mortalidad en los pacientes que ingresan a una UTI; sin embargo, lo más trascendente, es que de las múltiples intervenciones existentes; son mínimas las que han demostrado una mejora estadísticamente significativa en la sobrevivencia. De las intervenciones, la más importante es la implementación de un proceso de reanimación dirigido por metas dentro de las primeras seis horas de identificada la sepsis. (Myburg 2017) 


\section{Cristaloides y coloides en la reanimación del paciente crítico}

Vol. 3, núm. 3., (2019)

Gabriela Rosa Rodríguez Plaza; Néstor Ricardo Espinosa Sánchez; Renato Paolo Patiño Andrade; Marlon José Rivadeneira Rodríguez

Sin embargo, no existen los estudios suficientes para determinar la eficacia de los coloides en esta población. En el 2012, se llevó a cabo el estudio 6S (Scandinavian Starch for Severe Sepsis/Septic Shock) para evaluar la efectividad del hidroxietil almidón 130/0.4 comparado con solución ringer acetato en pacientes con sepsis grave y choque séptico. La mortalidad o dependencia de diálisis a los 90 días posteriores a la aleatorización ocurrió en el grupo de hidroxietil almidón en el 51 versus $43 \%$ del grupo de ringer acetato (RR 1.17, IC 95\% 1.01-1.36; p: 0.03). Los pacientes que requirieron de terapia de remplazo renal (TRR), en su mayoría fueron del grupo de hidroxietil almidón, asociándose a un incremento en la mortalidad a los 90 días (61 versus 44\% los que no recibieron TRR, p: <0.001).

En conclusión, la administración de hidroxietil almidón 130/0.42 en el proceso de reanimación de los pacientes con sepsis grave incrementa la mortalidad o dependencia de diálisis a los 90 días, con un número necesario a dañar de 13. El estudio CHEST (The Crystalloid versus Hydroxyethyl Starch Trial) evaluó la efi cacia y seguridad del hidroxietil almidón (130/0.4) comparada con la administración de solución salina $0.9 \%$ en el proceso de reanimación en pacientes en la UTI. La mortalidad a los 90 días fue de $18 \%$ en el grupo de hidroxietil almidón versus 17\% en el grupo de solución salina (RR 1.06, IC 95\% 0.96-1.18; p: 0.26). (Myllam 2016).

La probabilidad de sobrevivencia no fue significativa en ambos grupos (p: 0.27). La TRR fue implementada en el 7\% de los pacientes del grupo de hidroxietil almidón, comparada con el grupo de solución salina que fue del 5.8\% (RR 1.21, IC 95\% 1.00- 1.45; p: 0.04). Demostrándose entonces que, los niveles de creatinina incrementaron y los volúmenes urinarios disminuyeron de manera significativa en el grupo de hidroxietil almidón durante los primeros siete días (p: 0.004 y 0.003 respectivamente). (Myllam 2016). 


\section{Cristaloides y coloides en la reanimación del paciente crítico}

Vol. 3, núm. 3., (2019)

Gabriela Rosa Rodríguez Plaza; Néstor Ricardo Espinosa Sánchez; Renato Paolo Patiño

Andrade; Marlon José Rivadeneira Rodríguez

\section{Cristaloides y Coloides}

\section{Cristaloides}

Los fluidos se pueden agrupar en cristaloides y coloides. Los cristaloides son soluciones que contienen agua, electrólitos y/o azúcares en diferentes proporciones, y con respecto al plasma, pueden ser hipotónicos, isotónicos o hipertónicos. Su capacidad de expandir la volemia va a estar relacionada con la concentración de sodio, ya que es el factor que determina un gradiente osmótico entre los compartimentos extra e intravasculares. (Myllam 2016).

- Los cristaloides deben ser administrados primero en la reanimación del choque no hemorrágico (III).

- Las soluciones de AHE deben ser administradas con precaución en pacientes con bypass cardiopulmonar y sepsis (IIA).

- Los coloides se deben evitar o utilizar con precaución en pacientes con trauma craneoencefálico.

- La restricción hídrica es apropiada para pacientes hemo dinámicamente estables con Síndrome de Dificultad Respiratoria Aguda/Injuria Pulmonar Aguda (SDRA/IPA) (IIA); la combinación de coloides y diuréticos puede ser considerada en pacientes con SDRA/IPA hipo oncótica (III).

- Los coloides son preferidos para tratar la hipotensión relacionada con diálisis y mantener la hemo dinamia hasta obtener las metas de diálisis (IIA). 


\section{Cristaloides y coloides en la reanimación del paciente crítico}

Vol. 3, núm. 3., (2019)

Gabriela Rosa Rodríguez Plaza; Néstor Ricardo Espinosa Sánchez; Renato Paolo Patiño Andrade; Marlon José Rivadeneira Rodríguez

- La albúmina hiper oncótica debe ser administrada cuando se extraen grandes volúmenes de líquido ascitico en la ascitis refractaria a la terapia diurética (IIA).

- La albúmina puede ser administrada en unión con terapia antimicrobiana en pacientes con peritonitis bacteriana espontánea (IIA).

Corresponde entonces señalar que, se han desarrollado cristaloides con una composición más similar a la del plasma. Son las denominadas «soluciones balanceadas» (tabla 1). Las principales modificaciones que presentan son la reducción de las concentraciones de sodio y, sobre todo, de cloro, y la sustitución de este anión por lactato (Ringer Lactato) o por acetato, malato o gluconato (nuevas soluciones balanceadas). El pH de las mismas es menos ácido que el del suero salino, así como sus concentraciones de sodio y cloro son más similares a las del plasma. El efecto expansor de volumen que se consigue con estas soluciones es muy similar al del suero salino. (Myburg 2017).

Tabla $\mathbf{N}^{\circ}$ 2. Composición de los Cristaloides y comparación con el Plasma 


\section{Cristaloides y coloides en la reanimación del paciente crítico}

Vol. 3, núm. 3., (2019)

Gabriela Rosa Rodríguez Plaza; Néstor Ricardo Espinosa Sánchez; Renato Paolo Patiño

Andrade; Marlon José Rivadeneira Rodríguez

\begin{tabular}{|c|c|c|c|c|c|c|c|}
\hline Composición & $\begin{array}{l}\mathrm{NaCl} \\
0,9 \%\end{array}$ & $\begin{array}{l}\text { Ringer } \\
\text { Simple }\end{array}$ & $\begin{array}{l}\text { Ringer } \\
\text { Acetato }\end{array}$ & $\begin{array}{l}\text { Ringer } \\
\text { Lactato }\end{array}$ & $\begin{array}{l}\text { Plasma- } \\
\text { Lyte }^{2} 148\end{array}$ & Isofundin ${ }^{2}$ & Plasma \\
\hline $\mathrm{Na}^{-}, \mathrm{mmol} / \mathrm{I}$ & 154 & 147 & 130 & 131 & 140 & 145 & $135-145$ \\
\hline $\mathrm{Cl}^{-}, \mathrm{mmol} / \mathrm{I}$ & 154 & 155 & 112 & 112 & 98 & 127 & $98-105$ \\
\hline $\mathrm{K}^{-}, \mathrm{mmol} / \mathrm{l}$ & - & 4 & 5 & 5,4 & 5 & 4 & $3,5-5$ \\
\hline $\mathrm{Ca}^{2-}, \mathrm{mmol} / \mathrm{I}$ & - & 4 & 1 & 1,8 & 3 & 2,5 & 2,5 \\
\hline $\mathrm{Mg}^{2-}, \mathrm{mmol} / \mathrm{l}$ & - & - & 1 & & - & 1 & $1,5-2,5$ \\
\hline $\begin{array}{l}\text { Lactato, } \\
\mathrm{mmol} / 1\end{array}$ & - & - & - & 28 & - & & \\
\hline $\begin{array}{l}\text { Acetato, } \\
\mathrm{mmol} / \mathrm{l}\end{array}$ & - & - & 27 & & 27 & 24 & \\
\hline Otros, $\mathrm{mmol} / \mathrm{I}$ & - & - & & & $\begin{array}{l}\text { Gluconato } \\
23\end{array}$ & $\begin{array}{l}\text { Malato } \\
5\end{array}$ & $\begin{array}{l}\text { Bicarbonato } \\
24-28\end{array}$ \\
\hline $\begin{array}{l}\text { Osmolaridad } \\
\mathrm{mosm} / \mathrm{l}\end{array}$ & 308 & 309 & 276 & 277 & 295 & 309 & 291 \\
\hline $\mathrm{pH}$ & $\begin{array}{l}4,5- \\
7,0\end{array}$ & $5-7,5$ & $6,0-8,0$ & $5,0-7,0$ & $4,0-8,0$ & $5,1-5,9$ & $7,35-7,45$ \\
\hline
\end{tabular}

Fuente: (Myburg 2017)

Existen en el mercado 3 soluciones Ringer (Ringer simple, Ringer Acetato y Ringer Lactato). Hay que decir que el Ringer Simple no puede considerarse una solución balanceada por su contenido en sodio y cloro, muy similar al del suero salino. El más utilizado es el Ringer Lactato o solución de Hartmann, que es levemente hipo osmolar respecto al plasma y contiene $28 \mathrm{mEq}$ de lactato por litro, el cual es transformado en piruvato y, posteriormente, a bicarbonato durante su metabolismo como parte del ciclo de Cori. Esta cantidad de lactato figura como una mezcla de D-lactato y L-lactato. La forma L-lactato es la más fisiológica, siendo metabolizada por la lactato deshidrogenasa, mientras que la forma D-lactato se metaboliza por medio de la Da-deshidrogenasa. El Ringer Acetato no está comercializado actualmente en algunos países (Myburg 2017) 


\section{Cristaloides y coloides en la reanimación del paciente crítico}

Vol. 3, núm. 3., (2019)

Gabriela Rosa Rodríguez Plaza; Néstor Ricardo Espinosa Sánchez; Renato Paolo Patiño Andrade; Marlon José Rivadeneira Rodríguez

\section{Coloides}

Los coloides, por su parte representan partículas de alto peso molecular que atraviesan con dificultad las membranas capilares, de forma que son capaces de aumentar la presión oncótica plasmática y retener agua en el espacio intravascular. Producen efectos hemodinámicos más rápidos y sostenidos que los cristaloides, precisándose menos volumen que las soluciones cristaloides. Sin embargo, estos efectos parecen depender del contexto clínico: en sujetos hipovolémicos con presión capilar baja, la albúmina y los coloides sintéticos no tendrían ninguna ventaja hemodinámica sobre los cristaloides. Los coloides se dividen a su vez en sintéticos (gelatinas, almidones y dextranos) y naturales (albúmina). (Myburg 2017)

- Dextranos: Son una mezcla de polímeros de glucosa que están disponibles en 2 soluciones: dextrano 40 (peso molecular medio 40.000Da) y dextrano 70 (peso molecular medio 70.000Da). Se asocian con una incidencia considerable de efectos secundarios, tales como reacciones alérgicas, fracaso renal o diátesis hemorrágica, y prácticamente han caído en desuso en los últimos años.

- Gelatinas: Existen 2 formulaciones de gelatina: poligelina (gelatina unida por puentes de urea) y gelatina succinilada. Estas 2 formulaciones se diferencian no solo por las características químicas, sino también por su capacidad de expansión, composición electrolítica y episodios adversos. Tradicionalmente, el efecto adverso más temido con el empleo de las gelatinas es la posibilidad de reacción anafiláctica8, que ocurre en el 1\% con la poligelina y aproximadamente en el $0,1 \%$ con el uso de gelatina succinilada. El peso molecular de la gelatina succinilada es en torno a $30.000 \mathrm{Da}$, y a pesar de ello su 


\section{Cristaloides y coloides en la reanimación del paciente crítico}

Vol. 3, núm. 3., (2019)

Gabriela Rosa Rodríguez Plaza; Néstor Ricardo Espinosa Sánchez; Renato Paolo Patiño Andrade; Marlon José Rivadeneira Rodríguez

capacidad de expansión es similar a la del hydroxyethyl starch (HES, «hidroxietilalmidón») 130 (peso molecular 130.000Da). Recientemente se ha comercializado en España una nueva presentación con un menor contenido en cloro, que ha sido sustituido por acetato.

- Hidroxietilalmidón: Consiste en polímeros formados por polisacáridos naturales modificados. Se obtiene a partir del almidón de maíz o patata, mediante la sustitución de los grupos hidroxilos por grupos hidroxietiléter en las moléculas de glucosa de la amilopectina. Hay 2 características fisicoquímicas de interés que nos orientan sobre el comportamiento en el organismo de los HES: el peso molecular y el grado de hidroxilación, que se mide por el índice de sustitución molar. El índice de sustitución molar está determinado por el número de unidades de glucosa hidroxietiladas dividido por las unidades de glucosa presentes. A mayor número de unidades hidroxietiladas, mayor es el grado de sustitución y mayor la vida media de la molécula en el plasma.

- Albúmina: Como coloide natural, disponemos de las soluciones de albúmina que existen al 4 y al 20\%. En España y en general en Europa se utiliza preferentemente la presentación al 20\%, mientras que en Estados Unidos se emplea la del 4\%. Hay que decir que la presentación al $4 \%$ tiene un alto contenido en cloro $(120-130 \mathrm{mEq} / \mathrm{L})$, mientras que al $20 \%$ es una solución con bajo contenido en cloro $(20 \mathrm{mEq} / \mathrm{L})$. Las soluciones de albúmina se han utilizado para el tratamiento de pacientes críticos desde los años 40 . Sin embargo, debido a los resultados de un metaanálisis publicado en 1998 y que reportó un incremento de la mortalidad, el papel de la administración de albúmina en el paciente crítico se convirtió en un tema controvertido. Es bien conocido que la albúmina tiene 


\section{Cristaloides y coloides en la reanimación del paciente crítico}

Vol. 3, núm. 3., (2019)

Gabriela Rosa Rodríguez Plaza; Néstor Ricardo Espinosa Sánchez; Renato Paolo Patiño Andrade; Marlon José Rivadeneira Rodríguez

múltiples efectos fisiológicos que son muy relevantes en el enfermo crítico incluyendo la regulación de la presión coloidosmótica, el transporte plasmático de fármacos, la capacidad antioxidante y la modulación del óxido nítrico.

\section{Conclusiones.}

Dentro de las diversas opciones, el empleo de suero salino ha sido tradicionalmente la primera opción para la reanimación de los pacientes en shock. De hecho, ha sido elegido como el comparador en varios ensayos clínicos que han evaluado diversos coloides sintéticos o naturales en la reanimación de pacientes críticos Su contenido en sodio y cloro es ligeramente superior al del plasma y su empleo se ha asociado con acidosis hiperclorémica y probablemente con el desarrollo de fracaso renal

Si se infunden cantidades elevadas de solución salina, el excedente de cloro del líquido extracelular desplaza el bicarbonato, ocasionando acidosis hiperclorémica. Este efecto ha sido observado en pacientes posquirúrgicos $\mathrm{y}$ en politraumatizados, si bien parece que las consecuencias clínicas no son relevantes. Existe relación directa entre la cantidad de cloro administrada y la aparición de la acidosis metabólica.

Entiéndase con estas opiniones que, la acidosis metabólica que presentan los pacientes con sepsis grave y shock séptico es de etiología multifactorial, no obstante la acidosis hiperclorémica representa uno de los componentes predominantes, especialmente en los pacientes que fallecen. Recientemente se ha demostrado que la infusión de suero salino a voluntarios sanos ocasiona una reducción de la velocidad del flujo renal y de la perfusión de la corteza renal, lo cual no se observa con el empleo de una solución salina balanceada. 


\section{Cristaloides y coloides en la reanimación del paciente crítico}

Vol. 3, núm. 3., (2019)

Gabriela Rosa Rodríguez Plaza; Néstor Ricardo Espinosa Sánchez; Renato Paolo Patiño

Andrade; Marlon José Rivadeneira Rodríguez

Por consiguiente, se puede decir que los coloides son útiles para la reanimación, usados en combinación con los cristaloides cuando no están disponibles los hemoderivados. En estas circunstancias y teniendo en cuenta el costo, los coloides no proteicos son mejores que la albúmina, salvo cuando se desee una restricción de sodio o estén contraindicados los coloides no proteicos. En el choque distributivo no hemorrágico, los coloides no proteicos pueden emplearse cuando exista fuga capilar con edema pulmonar o periférico o tras la administración de 2 litros de cristaloides sin efecto alguno.

En definitiva, varias soluciones coloides han sido desarrolladas y usadas en la práctica clínica a nivel mundial. Su uso requiere del conocimiento de las propiedades químicas de los coloides y las características únicas de cada solución. Los coloides son costosos y presentan reacciones alérgicas y efectos adversos coagulopáticos que no son vistos con los cristaloides. Además algunas patologías críticas, cursan con aumento de la permeabilidad vascular, lo cual limita la habilidad de los coloides para mantener el espacio vascular y ejercer una presión osmótica adicional. La fuga vascular aumenta el coloide en el espacio intersticial, llevando a la formación de edemas.

En definitiva, se debe considerar que a pesar de algunas ventajas teóricas comparadas con los cristaloides, la administración de coloides no ha mostrado disminución del riesgo de injuria pulmonar aguda, o mejoría de la supervivencia. Las indicaciones precisas del uso de soluciones coloides incluyen estados hipoproteinémicos, pacientes que requieren expansión plasmática y no son capaces de tolerar grandes cantidades de volumen, procedimientos reconstructivos y ortopédicos que requieran prevención de formación de trombos y leucoféresis. 


\section{Cristaloides y coloides en la reanimación del paciente crítico}

Vol. 3, núm. 3., (2019)

Gabriela Rosa Rodríguez Plaza; Néstor Ricardo Espinosa Sánchez; Renato Paolo Patiño Andrade; Marlon José Rivadeneira Rodríguez

\section{Bibliografía.}

Arias, F. (2010). Paradigmas de la Investigación Científica. España: Editorial: Luces.

Beck, G. (2016). The immunological effects of hypertonic saline. Review Medical Critic de Nueva York, 12 - 22.

Brattong, D. (2016). Intravenous fluid therapy in neurologic injury. Revista Panamericana de Salud de la Universidad Nacional de Medellín en Colombia, 8 - 13.

Dávila, N. (2012). Paradigmas de la Investigación Científica. Pereire, Colombia: Editorial: Las Brisas.

Lang, H. (2015). Effects of albumin and Ringer's lactate on production of lung cytokines and hydrogen peroxide after resuscitated hemorrhage. Review: Critic Care Medc, 22 - 30.

Moss, P. (2017). Beneficios que brinda los coloides y Cristaloides a la reanimación en pacientes Críticos. Revista de Medicina Intensiva de la Universidad de la Habana en Cuba, 7 - 13.

Myburg, S. (2017). Reanimación de pacientes Críticos a través de Fluídos. Informe Sanitario de medicina Intensiva, 15.

Myllan, C. (2014). Coloides y Cristaloides, Características Clínicas . Revista Médica Informativa de la Universidad Nuevo México en México, 33 - 35.

Nicholson, A. (2016). Efectos de los Cristaloides y oloides en pacientes Críticos. Revista Médica de la Universidad deBuenos Aires en Argentina, 6 - 14.

Rozoli, M. (2015). Fluid Management: The Pharmacoeconomic dimension Critice. The Journal Medicine Critice, 32. 